ISSN 1984-3755

Licenciado sob uma Licença Creative Commons

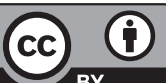

\title{
Actuosa Participatio: il vero fine della riforma liturgica
}

\author{
Participação ativa: o verdadeiro propósito da reforma litúrgica
}

\begin{abstract}
Andrea Grillo
Doutor em Teologia pelo Instituto Teológico de Pádua, Itália; mestre em Liturgia pelo mesmo Instituto, pelo qual já se graduara em Teologia; professor no Pontifício Ateneu Santo Anselmo, em Roma, no Instituto de Liturgia de Pádua (Abadia de Santa Justina) e no Instituto Teológico de Ancona - Itália, e-mail: grilloreba@gmail.com
\end{abstract}

\section{Riassunto}

Il saggio indica alcune idee sbagliate presente nella coscienza ecclesiale in relazione ad una delle questioni più delicate e appariscenti della riforma liturgica del Vaticano II. Senza affrontare loro, con l'aiuto del metodo storico è il metodo da teologica, riforma liturgica fissato dal Consiglio rimangono incompleti e persino controproducente. Infatti, la partecipazione attiva dei fedeli alla liturgia - che è fonte e culmine della vita ecclesiale - non è qualcosa di accidentale o superfluo, ma essenziale, in quanto il "Le azioni liturgiche non sono azioni private ma celebrazioni della Chiesa Qual è il 'sacramento di unità', cioè del popolo santo "e" appartengono all'intero corpo della Chiesa e manifesto e affetto ", anche se raggiungere" i singoli membri in modo diverso, come la diversità degli ordini ministeri e la loro partecipazione effettiva "(SC 26). Il tema della partecipazione attiva implica quindi una riformulazione di tutta la strada di sentire e di agire nella sfera religiosa, il Consiglio, salvataggio del Nuovo Testamento e paradigma 
patristico da un lato, e moderna cristiana autocoscienza del soggetto, l'altro assume come prospettiva. Da qui la necessità di chiamare alla coscienza il complesso rapporto tra popolazione attiva e la riforma liturgica, in modo che le sue prestazioni istituzionali e pastorali, non solo calcio, ma i progressi costanti.

Parole-chiave: Consiglio. Riforma liturgica. Partecipazione. Oggetto.

\section{Resumo}

O artigo chama a atenção para alguns equívocos presentes na consciência eclesial em relação a um dos temas mais sensíveis e vistosos do Concílio Vaticano II: a reforma litúrgica. Sem enfrentá-los, com a ajuda seja do método histórico seja do método teológico, a reforma litúrgica definida pelo Concílio continuará incompleta e até mesmo contraproducente. Com efeito, a participação ativa dos fiéis na liturgia - que constitui a fonte e o cume de toda a vida eclesial - não é algo acidental ou dispensável, mas essencial, uma vez que "as ações litúrgicas não são ações privadas, mas celebrações da lgreja, que é o 'sacramento da unidade', isto é, o povo santo" e "pertencem a todo o corpo da Igreja, e o manifestam e afetam", ainda que atinjam "a cada um dos membros de modo diferente, conforme a diversidade de ordens, ministérios e da participação atual" (SC 26). O tema da participação ativa implica, portanto, uma reformulação de todo o modo de sentir e de agir no âmbito religioso, que o Concílio, resgatando o paradigma neotestamentários e patrístico, de um lado, e a autoconsciência do sujeito cristão moderno, do outro, assumiu como perspectiva. Daí a necessidade de se chamar à consciência a complexa relação entre a participação ativa e a reforma litúrgica, de modo que a sua atuação institucional e pastoral não só não retroceda, mas avance coerentemente.

Palavras-chave: Concílio. Reforma litúrgica. Participação. Sujeito.

La recente riflessione sul tema della "partecipazione attiva" ha messo in luce una serie di equivoci niente affatto secondari, che si sono come annidati nella coscienza ecclesiale e senza la cui soluzione ogni Riforma Liturgica rimarrà sempre incompiuta o addirittura controproducente. Nel dipanare tale ardua questione, che interessa obiettivamente non solo la 
teoria teologica, ma anche la pratica pastorale, il metodo sistematico e il metodo storico debbono aiutarsi reciprocamente. Scienze umane (tutte) e scienze teologiche (tutte) pongono in luce il profilo decisivo della questione e le sue conseguenze sul corpo ecclesiale e sulla qualità spirituale della vita cristiana. Per questo dobbiamo precisare anzitutto due aspetti del nostro oggetto:

a) la evidenza del bisogno di "partecipazione attiva, consapevole, pia, comunitaria..." emerge soprattutto nell'”ancien régime rituale". Le pagine più alte sul nostro tema sono state scritte - inevitabilmente - sotto la vigenza del "rito di Pio V", frutto del concilio tridentino. Questo, tuttavia, non deve far pensare incautamente che sia il rito di Pio V a garantire la partecipazione attiva. Anzi, questo appare proprio come un pericoloso paralogismo - un vero "blocco mentale" - privo di fondamento razionale e profondamente tendenzioso - quasi l'indice di una strutturale autoimplicazione fallace della argomentazione ${ }^{1}$. Mentre deve essere dedicata maggiore attenzione al fatto che - nonostante il rito cosiddetto "pre-conciliare"- anzi, proprio in rapporto a quel rito, tutti i maggiori liturgisti della prima metà del XX secolo abbiano saputo e potuto elaborato la più profonda riflessione su quelle elementari esigenze di conoscenza/comunicazione, di azione/passione e di emozione ${ }^{2}$, che avrebbero poi motivato e determinato la Riforma Liturgica.

b) Questa prima affermazione indica che tra l'esigenza di partecipazione attiva e la prassi di Riforma c'è una relazione complessa e non univoca, che può anche diventare "ostacolo" o "impedimento" alla vita liturgica. Il regime dei riti precedenti ha suscitato un profondo ripensamento delle modalità con cui il cristiano "prende

1 Di tale errore logico sembrano compiacersi tutti quei "laudatores temporis acti” che hanno smarrito, oltre alla memoria, anche il senso del limite e che pertanto si illudono di poter salvaguardare la tradizione mediante un pericoloso compromesso tra disperazione e presunzione. Esemplare per mancanza di rigore e per approssimazione è il volumetto sgangherato di BUX, N. La riforma di Benedetto XVI. Casale Monferrato: Piemme, 2008, compresa la disinformatisima "Presentazione", inultilmente elogiativa, del giornalista, aspirante teologo, Vittorio Messori.

2 Una intelligente rilettura della "participatio consapevole, actuosa, pia" in rapporto alle tre "facoltà" (del conoscere, del volere e del sentire) è stata proposta di recente da BONACCORSO, G. I principali orientamenti dello studio della liturgia. In: CARR, E. (Ed.). Liturgia opus trinitatis: epistemologia litúrgica. Roma: Pontificio ateneo S. Anselmo, 2002. p. 13-25. (=Studia Anselmiana 133, Analecta liturgica, 24). 
parte" alla liturgia, mentre il mutamento dei riti stessi è come se avesse attutito e quasi reso più opaca, ottusa e meno determinata la riflessione teologica, sottraendo vieppiù alla prassi delle "Liturgie riformate" il supporto teorico e il fine ultimo della loro legittimazione. Fino al punto che, proprio la abitudine ai "nuovi riti" ha di fatto reso meno evidente il fine ultimo degli stessi, ossia la esigenza che si possa "prendere parte" all'unica azione rituale da parte di tutto il popolo di Dio, sia pure a diverso titolo e con diverse forme di ministero.

Questo significa che il nostro procedere dovrà recuperare l' evidenza della "partecipazione attiva come fine ultimo della Riforma", per comprendere di nuovo l'atto riformatore in tutta la sua storica necessità, ma anche in tutta la sua strutturale insufficienza $a^{3}$. Per farlo esso dovrà "ricominciare dall'inizio", ossia da due progetti di ripensamento della "partecipazione" degli anni 30 e 50 (§. 1), per poi studiare meglio la differenza paradigmatica tra MD e SC (§. 2), approdare agli imbarazzi attuali (§. 3), con tutta la falsa nostalgia di cui si alimentano sconsideratamente e con tutta la apparente continuità che cercano di procurare, per indirizzare la domanda fondamentale circa la "actuosa participatio" verso una soluzione plausibile.

\section{La riflessione preconciliare (in P. Parsch e G. Lercaro)}

Vorrei soffermarmi anzitutto su due autori "minori" (come P. Parsch e G. Lercaro), dai quali possiamo trarre con bella chiarezza il senso della domanda di "actuosa participatio", come un elemento che non deriva da una considerazione estrinseca (sociologica, antropologica, estetica) del rito , ma che scaturisce piuttosto dal suo legame oserei dire ontologico e teologico con il rito. E tuttavia, essendo i riti "ontologicamente contingenti", ecco che per recuperarne il senso - pastoralmente - occorre anche e anzitutto una competenza

3 E' urgente oggi ricordare e come rimeditare le parole lucide di A. Bugnini, quando ricordava che: "la partecipazione e l'inserimento del popolo di Dio nella celebrazione liturgica sono lo scopo ultimo della riforma, sono stati l'obiettivo del movimento liturgico". BUGNINI, A. La riforma liturgica (1948-1975). Roma: CLV - Edizioni Liturgiche, 1997. p. 21. 
sociologica, psicologica, antropologica ecc. In questa sorprendente dinamica e tensione - che non impedisce affatto di parlare della essenza dei riti, ma che esplicitamente si oppone ad ogni possibile configurazione di "riti essenziali" - hanno lavorato con gusto e con intelligenza questi due uomini, che si sono rivelati di grande importanza per il Movimento Liturgico europeo.

\section{Giacomo Lercaro e il libretto “A messa figlioli!”}

La lettura di questo interessante libretto dell'Arcivescovo di Bologna (LERCARO, 1955) ${ }^{4}$ suona per i cristiani di 55 anni dopo come una sorprendente e benefica provocazione. Vorrei quasi dire che - pur provenendo dalle nebbie del pre-concilio e non potendo avere il Concilio altrimenti che al di là di se stesso - questo libro è salutare e ci aiuta a guarire da una grave limitazione - e da una certa limitatezza - della coscienza liturgica attuale. Ossia dal fatto che la nostra coscienza risulta essere stata viziata dal Concilio e dalla Riforma liturgica ad esso conseguente.

No, il libretto del Cardinal Lercaro non corre ancora il nostro pericolo. Deve anzi "lavorare" con una condizione per certi versi opposta alla nostra. Potrei cercare di definire tale condizione con queste parole: mentre noi abbiamo riti nuovi, ma rischiamo di rivolgerci ad essi con atteggiamenti vecchi, Lercaro aveva necessariamente a che fare con riti vecchi, che cercava di rileggere e rivivere con atteggiamenti nuovi. Lercaro, in altri termini, viveva allora immerso in quello che era il Movimento Liturgico, senza avere ancora la Riforma liturgica come un "dato di fatto" acquisito e pacifico, mentre noi percepiamo la Riforma Liturgica come cosa ovvia e scontata, ma abbiamo largamente perso lo spirito del Movimento liturgico. In verità non dovremmo mai dimenticare che Casel, Guardini, Parsch, Vagaggini hanno vissuto quasi tutta la loro esistenza prima del Concilio!

Se delle conseguenze pastorali di questa difficoltà non avrò qui il tempo di parlare, vorrei però almeno scavare storicamente e teologicamente sul

4 Interessante è il raffronto tra questo testo e il successivo, frutto di predicazione ad un corso di esercizi (19651966) per i presbiteri e i diaconi dell'Archidiocesi bolognese: LERCARO, G. L'eucaristia nelle nostre mani: liturgia e catechesi. Bologna: EDB, 1968. 
fondamento di questa "impasse", che tanto ci affatica e ci mette alla prova. Articolerò il mio discorso in pochi passi, dai quali emergerà una rilettura dei contenuti del libretto "A messa, figlioli!" sullo sfondo del Movimento Liturgico, con tutto il gusto per la "actuosa participatio" (benintesa, ovviamente), che trova realtà nella grande sintesi conciliare e nella eredità che del Concilio è stata vissuta (spesso assai unilateralmente) in termini di "sola Riforma"

Ora a me pare che il libretto del Card. Lercaro costituisca da questo punto di vista un'ottima prova di questa tendenza originaria del Movimento Liturgico, che noi abbiamo talora dimenticato e spesso anche esplicitamente negato. Rileggeremo dunque il testo per tornare all'altezza di questa grande concezione e della persona che ha pensato, lottato e pregato per questo nobile progetto.

Il primo punto dal quale dobbiamo partire è la coscienza che il Movimento Liturgico si è sviluppato non come una iniziativa estemporanea che modificava evidenze acquisite della tradizione ecclesiale, bensì come il tentativo di dare risposta ad una questione nuova nella storia della Chiesa, ossia alla domanda: che ruolo hanno i riti nel costituirsi della esperienza di fede ecclesiale? A questa domanda l'intellettualismo, il dottrinalismo e il razionalismo - ma anche il positivismo moderno, compreso quello teologico - non sapevano più rispondere. Di qui la crisi della esperienza liturgica, che comincia nella tarda modernità, e di cui ci si accorge a partire dal XIX secolo. La questione si è aperta dunque senza i liturgisti: i liturgisti sono nati per cercare di risolvere il problema, per ridare anche all'uomo tardo moderno la necessaria risposta alla domande sul "perché celebrare?" e sul "che cosa celebrare?" e non più soltanto sul "come celebrare?".

Ciononostante, tra le cose più sorprendenti della recente fase di recezione della nuova coscienza liturgica, si notano le serpeggianti forme

\footnotetext{
5 Un principio non scritto del "sola riforma" vale un poco come il classico principio protestante del "sola Scriptura", ma - come quello - non risolve affatto il problema del rapporto con la tradizione, che non si lascia mai ridurre ad un principio testuale, sia esso biblico oppure liturgico. Alla "questione liturgica" non si potrà mai rispondere con una "pura Riforma", e questo fa parte della quintessenza del Movimento Liturgico stesso, che ha cercato anzitutto di ritrovare le ragioni di una "partecipazione comune all'unico atto liturgico" (questo è il senso più autentico di ciò che chiamiamo "partecipazione attiva") da guadagnare anche con il nobile strumento della Riforma dei riti. La Riforma, in altri termini, non è altro che uno strumento necessario - ma mai sufficiente - per far sì che la liturgia torni ad essere fons di tutta l'azione della Chiesa.
} 
di nostalgia liturgica del preconcilio. Ma dobbiamo ricordare che 50 anni fa un uomo come il Card. Lercaro poteva cominciare dalla costatazione della crisi in cui versava la partecipazione alla liturgia nel suo tempo.

Raccontando un episodio di incontro con un Arcivescovo di rito siro-malabarico, egli diceva infatti: "ebbi netta la sensazione del disagio che debbono provare i nostri fedeli di fronte alla nostra Messa, quando essa resti per loro un oscuro susseguirsi di parole incomprensibili e di gesti misteriosi" (LERCARO, 1955, p. 16).

Movimento Liturgico, Concilio e Riforma liturgica sono il principio del rimedio, non la causa del male. Il capovolgimento del rapporto tra causa/ effetto (e il paralogismo post hoc ergo propter hoc) sembra oggi la scorciatoia per non capire più il fenomeno al quale G. Lercaro si era appassionato e quindi per non capire neppure più nulla del suo effettivo ed efficace magistero.

Tuttavia non basta denunciare la crisi. Occorre recuperare il senso più autentico del celebrare rituale e trovare un nuovo "approccio" al momento liturgico come momento originario del rivelarsi di Dio e della risposta che l'uomo presta nella fede. Il "senso teologico della liturgia", la sua carica "spirituale" e la sua importanza per il costituirsi dell'esperienza ecclesiale sono affermazioni che grazie al Concilio Vaticano II, alla Riforma liturgica e alla prassi di culto rinnovata, sono quasi diventate "luoghi comuni" della coscienza ecclesiale odierna. Ma una visione puramente giuridico-rubricistica dei riti cristiani era ancora negli anni 30 o 40 del XX secolo del tutto ovvia. La liturgia non aveva nessuno spessore teologico, alcuna potenzialità spirituale e stava semplicemente a valle della istituzione ecclesiale, come una sua "protestatio" esterna e esteriore.

Ad esempio, dedicarsi al "precetto domenicale" poteva al massimo identificarsi come una delle conseguenze della fede, ma non aveva nulla a che fare con la fede stessa. G. Lercaro sapeva invece di questa originarietà elementare dell'atto celebrativo eucaristico:

Quando le nostre popolazioni avranno compreso che cosa è la Messa e la sentiranno non più come un'azione incomprensibile e quasi magica del sacerdote, ma invece come cosa loro, nella quale hanno parte e donde ricevono un ricco apporto di vita spirituale, sarà facile persuaderle della doverosità di adempiere il precetto: più che un precetto, anzi, la Messa festiva diventerà un bisogno dello spirito, come è, per il corpo, il pasto quotidiano (LERCARO, 1955, p. 17). 
In questa espressione semplice di Lercaro vi è tutta la coscienza delle dimensioni che la liturgia sa mettere in gioco e plasmare, con il suo stile e il suo linguaggio particolare. Una visione ecclesiale di comunione, una esperienza spirituale e il superamento di una mentalità del "minimo necessario" si sposano alla perfezione in queste poche righe.

Per questo motivo è possibile che un Arcivescovo, nel 1955, scrivesse un Direttorio per la partecipazione attiva dei fedeli alla s. Messa letta (così suona il sottotitolo del libretto che stiamo analizzando). In tale partecipazione si giocava a suo avviso non un dettaglio marginale tra i doveri del cristiano, ma l'avvenire della Chiesa stessa!

\section{Pius Parsch e il concetto di partecipazione alla liturgia}

Nella prospettiva di P. Parsch, già 30 anni prima di Lercaro, a Vienna, il Movimento Liturgico non poteva che tradursi in "Movimento Liturgico popolare". E' l'oggetto stesso (meglio, il soggetto stesso della sua ricerca, il "Cristo totale, capo e corpo" di cui parlerà ufficialmente 20 anni dopo l'enciclica di papa Pio XII "Mediator Dei") ad imporre questa evoluzione interna alla storia del Movimento Liturgico. Credo che questo sia un punto che ci permette di riqualificare adeguatamente anche il valore quasi profetico del lavoro teologico svolto nella prima metà del secolo scorso da P. Parsch: ossia, quello di recuperare un rapporto significativo tra "metodo" e "oggetto", tra forma e contenuto della scienza liturgica. Mi spiego subito più chiaramente.

Il contenuto di una rinnovata scienza liturgica poteva ben essere stato il frutto di una serie di lunghe indagini filologiche, storiche, archeologiche, teologiche, patristiche, bibliche: ma se tale ritorno alle fonti della liturgia non aveva come meta il ritorno alla liturgia come fonte, tutto sarebbe apparso inutile, accessorio, illusoriamente elitario. Da questo punto di vista, Parsch è stato un fine teorico del Movimento Liturgico, perché ha visto, molto pirma di tutti gli altri, il pericolo di un ressourcement che non avesse come fine primario la riscoperta della liturgia come fons.

Potremmo quasi dire: il concetto di liturgia è tale che non può essere soltanto concetto, ma azione, opera, funzione. Essa sporge fuori 
dall'intellettualismo delle definizioni e preme verso una realizzazione simbolico-rituale di ciò che la Chiesa pensa di sé. Per questo il rapporto tra concetto e azione costituisce il cuore di un ripensamento sistematico che si traduce, per P. Parsch, immediatamente, nella esigenza di condurre tutti i battezzati e non solo i ministri ordinati - ad una piena soggettività liturgico-ecclesiale.

Ciò corrisponde a quella esigenza motodologica ed epistemologica che possiamo considerare come una delle acquisizioni più lucide del Movimento Liturgico del XX secolo, che di recente è stata espressa in modo esemplare da G. Bonaccorso:

Il sapere (liturgico) è interno al suo oggetto, con la conseguenza che il modo della riflessione teologica viene elaborato, almeno in parte, all'interno e, quindi, secondo il modo della prassi liturgica: il metodo (teologico) è interno all'oggetto litúrgico (BONACCORSO, 2002, p. 97).

Solo a patto di poter ripetere con $\mathrm{M}$. Blondel la bella sentenza secondo cui: "nella pratica religiosa è necessario che i rapporti ordinari tra il pensiero e l'azione siano al tempo stesso conservati, completati e rovesciati" (BLONDEL, 1993, p. 526), potremo davvero comprendere in che senso è possibile sostenere, con pienezza di senso, questa bella asserzione con cui P. Parsch definisce la liturgia come "principio formale" della Chiesa:

La liturgie est donc la plus haute manifestation de la vie de l'Eglise; elle est à la fois le visage et la bouche de l'Eglise. Ce que l'Eglise sent, pense, met en oeuvre, elle le manifeste dans la liturgie. Elle est exactement le principe formel de l'Eglise (PARSCH, 1950, p. 21).

Per tutto ciò, e alla luce di quanto precede, è possibile comprendere in che senso la liturgia sia già, per Parsch, pienamente fons et culmen, come poi dirà con luminosa chiarezza Sacrosanctum Concilium. Ma questo sottintende una elaborazione teorico-pratica del rapporto con la liturgia in cui la partecipazione attiva e il sacerdozio universale siano non profili eventuali ma fondamenti strutturali della esperienza ecclesiale del discepolato di Cristo ${ }^{6}$, e per questo vadano perseguiti come priorità per conseguire - attraverso la Riforma della liturgia - la Riforma della Chiesa!

6 Un'utile rilettura delle principali questioni teologiche legate al ruolo strutturale della "partecipazione attiva". 
Oggi questa lucidità di P. Parsch sembra mancare a molti settori teologici e a diversi ambienti ecclesiali. Ed è per questo che lo studio del pensiero di Parsch ${ }^{7}$, pur provenendo da prima della Riforma, può permettere di rileggerne lo spirito e i contenuti con lucidità e e sensibilità profetica, illuminando adeguatamente il concetto centrale di partecipazione e le sue "difficoltà" attuali.

\section{Le evidenze conciliari (ossia la differenza "paradigmatica" tra MD e SC)}

Chiarito il primo passaggio, vorrei brevemente approfondire il senso della "differenza" tral'enciclica Mediator Dei e la Costituzione Conciliare Sacrosanctum Concilium proprio nel cuore della lettura della nozione di "partecipazione". Pur con tutte le oscillazioni di significato, il passaggio decisivo consiste nell'assumere il rito come "linguaggio comune della Chiesa", che non lascia nessuno all'esterno e che non sopporta alcun spettatore. Occorre però qui - implicitamente - sia una nuova nozione di rito, sia un nuovo concetto di teologia.

Il cuore della novità sta nel delicato rapporto tra una visione classica di "partecipazione" - che risulta ormai insufficiente - e una visione nuova che intuisce la profondità del rito litrugico per la vita di fede. In effetti, per lunghi secoli la coscienza teologica ecclesiale circa la liturgia - e segnatamente circa la eucaristia - è rimasta per così dire "bloccata" dalla preoccupazione prioritaria di una contrapposizione confessionale: antiprotestantesimo cattolico e anticattolicesimo protestante facevano il paio nel comprendere se stessi quasi soltanto come valorose opposizioni ad un "nemico" pericoloso.

L'eco di tale posizione controversistica rimane ancora fortemente presente nella stessa Mediator Dei (ai nn. 53-115, sul “culto eucaristico"),

si può trovare bene illustrata nei due recenti saggi: CARDITA, A. Actuosa participatio: reflexão à volta de uma noção chave na "questão litúrgica". Humanística e Teologia, v. 25, p. 87-104, 2004; DE CLERCK, P. La participation active. Perspectives historico-liturgiques de Pie X à Vatican II. Questions Liturgiques, v. 85, n. 1/2, p. 11-29, 2004.

7 Per un aggiornamento bibligrafico completo sull'autore rimando all'accurata rassegna: REDTENBACHER, A. Pius Parsch in der Liturgiewissenschaftlichen Rezeption. Veröffentlichte und Unveröffentlichte Arbeiten über Pius Parsch. Heiliger Dienst, v. 58, p. 142-167, 2004. 
costituendone un limite obiettivo, per quanto ormai i frutti del Movimento Liturgico fossero quasi del tutto maturi e si imponessero già largamente sul clima riduttivo della apologética ${ }^{8}$.

In particolare, la "partecipazione dei fedeli" è compresa come "intimo contatto" dell'anima con il senso della celebrazione, ma ci si premura subito di escludere una semplice parificazione tra laici e clero come anche una minore validità delle "messe che si celebrano in privato", consigliando addirittura in conclusione una sorta di "parallelismo devozionale" (per i rudes) rispetto al rito eucarístico ${ }^{9}$. Questa è la condizione da cui si parte per la nozione di "partecipazione" e nella quale irrompe la nuova prospettiva di SC, per quanto preparata già dalla evoluzione stessa del pensiero di papa Pio XII.

Il cambiamento di prospettiva introdotto da SC consiste, essenzialmente, nell'aver spostato decisamente le priorità del discorso sulla eucaristia. L'elemento più qualificante di questa novità sta nella riscoperta e rivalutazione della stretta correlazione fra diversi livelli del discorso e della esperienza, che nel regime precedente (e, purtroppo, anche in parte di quello successivo e attuale) sono rimasti separati e giustapposti. Nello specifico SC propone una lettura non accessoria della partecipazione attiva da parte dei fedeli e la forma specifica di tale partecipazione.

Proprio su questo ultimo punto notiamo le più grandi differenze rispetto al passato e, forse, anche rispetto ad oggi! In effetti il testo, al n. 48 riguardo al mistero eucaristico, si esprime così: "per ritus et preces id (=eucharisticum mysterium) bene intelligentes", ossia formula

8 Per una rilettura in questo senso cf. CATELLA, A. Dalla Costituzione conciliare "Sacrosanctum Concilium" all'enciclica "Mediator Dei". In: AA. VV. La Mediator Dei e il Centro di Azione Liturgica: 50 anni alla luce del movimento liturgico (Bibliotheca "Ephemerides Liturgicae" - Sectio Pastoralis, 18), C. L. V. Roma: Edizioni Liturgiche, 1998. p. 11-43.

9 II concetto di partecipazione dei fedeli in Mediator Dei è essenzialmente legato non al rito, ma allo stato d'animo (cf. n.67). Così, coloro che hanno difficoltà ad entrare nella logica liturgica e non vogliano rinunciare a partecipare al sacrificio eucaristico "possono certamente farlo in altra maniera... come, per esempio, meditando piamente i misteri di Gesù Cristo, o compiendo esercizi di pietà e facendo altre preghiere, che pur differenti nella forma dai sacri riti, ad essi tuttavia corrispondono per la loro natura" (n. 90). Questo consiglio esplicito di parallelismo partecipativo di fatto non riesce ancora ad immaginare la novità che SC introdurrà, rendendo inseparabile forma rituale e partecipazione attiva. In questo sta proprio la novità più significativa che il Concilio ha saputo recuperare e autorevolmente riproporre.

Rev. Pistis Prax., Teol. Pastor., Curitiba, v. 4, n. 2, p. 441-461, jul./dez. 2012 
ufficialmente la insufficienza di una partecipazione della sola anima, con cui prima (e poi) ci si è "difesi" dal Movimento Liturgico e dal senso della Riforma da esso promossa.

E' evidente che il vero motivo della Riforma consiste dunque nella possibilità di intelligere il mistero eucaristico proprio per ritus et preces, ossia nella partecipazione consapevole, pia e attiva alla azione liturgica. E' dunque la azione il modo primario della intelligenza liturgica. Con questa consapevolezza non è il significato nell'anima (actus animae) il "primum", ma è piuttosto il significante e l'atto corporeo (usus rerum exteriorum) ad essere messo in primo piano. La Riforma del rito eucaristico è perciò motivata essenzialmente dalla esigenza di recuperare appieno e per tutti questo livello rituale e orante della intelligenza eucaristica. Non si tratta, in altre parole, di una Riforma al servizio della solita comprensione intellettuale, ma abbiamo piuttosto a che fare con un mutamento prospettico e con un recupero esperienziale in vista di un nuovo e originario modo di comprendere la verità della eucaristia, in equilibrio tra sensibilità e intelletto. E' la rituum forma (SC 49), la forma rituale, ad assicurare la piena efficacia pastorale del sacrificio eucaristico.

Questa prospettiva cambia anche inevitabilmente il tono generale con cui si parla delle diverse questioni: l'approccio in termini di actio sacra si preoccupa di recuperare anzitutto la pienezza del gesto rituale, piuttosto che la integralità del significato ${ }^{10}$. La considerazione della pienezza della partecipazione concentra la attenzione sull'atto piuttosto che sul significato e ciò modifica profondamente anche il metodo della teologia della eucaristia. Prevale il "positivo" rispetto al "negativo", il "propositivo" sul "difensivo", l'articolato sull'essenziale: in altri termini, per la nuova mentalità introdotta dal Concilio - ma lungamente preparata dal Movimento Liturgico - l'accidente non è irrilevante per la sostanza: potremmo dire che nell'eucaristia, grazie a queste nuove considerazioni, si "riscopre" che gli accidenti non sono accidentali.

Ciò, come abbiamo detto, modifica nello stesso tempo la teologia e la prassi eucaristica, poiché supera quella logica del "minimo necessario"

${ }^{10}$ Qui appare chiaramente il prevalere dell'approccio liturgico-sacramentale su quello dogmatico-disciplinare e/o soggettivo-spirituale. Alla logica del "minimo necessario" si sostituisce quella del "massimo gratuito". 
rituale, che per il primato dell'anima poteva/doveva essere sufficiente, ma che nella nuova prospettiva non basta più, anzi diventa continuamente fuorviante e principio di gravi incomprensioni.

Per questa prospettiva il vero e gravissimo abuso eucaristico è costituito dalla riduzione della eucaristia al suo minimo, alla sua essenza, al suo scheletro concettuale. Di qui nasce anche il bisogno urgente di una piena articolazione spazio-temporale della azione eucaristica, che recuperi tutta la ricchezza del riferimento biblico, dell'omelia, della preghiera dei fedeli, di una lingua comprensibile, della comunione con pane e calice, dell'unità della celebrazione e della possibilità di concelebrazione. Si noti bene: non uno di questi elementi è tradizionalmente ad necessitatem, ma tutti sono ad solemnitatem. Nella vecchia prospettiva questi sono tutti aspetti dispensabili e in certo modo contingenti, accessori, accidentali rispetto al significato teologico della eucaristia. Ora, invece, poiché è l'azione liturgica - e non semplicemente il suo significato - il centro della relazione eucaristica, ogni sua "parte" è atto simbolico-rituale qualificante teologicamente l'eucaristia. La modificazione che questo nuovo senso delle priorità comporta sia sul piano della esperienza pastorale-esistenziale, sia sul piano della riflessione teologico-concettuale, non è ancora del tutto entrato nella consapevolezza ecclesiale.

Se l'autorevole magistero liturgico di G. Lercaro e di P. Parsch - 50 o 70 anni fa - era già entrato pienamente e con largo anticipo - sai pure a suo modo - nella logica simbolica e rituale di questa azione liturgica, questo non è il caso del nostro tempo, nel quale proprio su questo aspetto notiamo spesso casi di vera e propria regressione.

\section{I problemi del postconcilio (la necessaria mediazione tra differenti "stili" e "metodi" di diversi "paradigmi")}

Le questioni fondamentali che si sono aperte dopo il Concilio Vaticano II derivano essenzialmente dal mancato coordinamento di questi due fronti - tradizionalmente basati su un rapporto presupposto, ma che ora diventa facilmente rapporto rimosso: ossia tra una nuova nozione di rito e un nuovo metodo nel riferirsi al "dato" da parte del lavoro teologico. 
Spesso è accaduto che le nuove teologie lavorassero con una nozione vecchia di rito (il vecchio "ritus servandus"), mentre le nuove coscienze rituali elaboravano teorie sulla base di concezione vecchie della teologia (o positiva o speculativa). Il "nuovo paradigma" conciliare è nuovo proprio perché pretende - nello stesso tempo - un aggiornamento delle dottrine teologiche e delle esperienze fondamentali, senza poter mai raggiungere alcun risultato convincente se non procede su entrambi i fronti, coerentemente e parallelamente. D'altra parte, come è ovvio, se non si permette alla nuova comprensione di rito e al nuovo approccio teologico di affermarsi in concreto, esso resterà sempre un semplice lessico nuovo cui corrisponderà nei fatti il permanere del canone precedente, che, alla lunga, reimporrà il suo canone sulla partecipazione, sul ministero, sull'essenza del sacramento ecc. ecc.

Qui rimando al gioco di paradigmi di cui ho trattato altrove (GRILLO, 2008). D'altra parte mi pare che vi siano nel dibattito attuale due posizioni sostanzialmente dissonanti, una delle quali valorizza profondamente le acquisizioni del ML (Bonaccorso), mentre una seconda guarda sostanzialmente con sospetto proprio al superamento della nozione preconciliare di "partecipazione" (Ratzinger). Un breve esame di queste due posizioni "esemplari” mi pare utile a questo punto della mia ricerca, proprio con funzione sintetica.

\section{La "partecipazione attiva" secondo G. Bonaccorso}

In un recente saggio (BONACCORSO, 2009), G. Bonaccorso puntualizza accuratamente il senso della nozione di "actuosa participatio" come emerge dalla elaborazione del ML. In esso si possono rilevare alcune affermazioni di grande lucidità intorno al ruolo "strategico" di tale nozione come "scopo ultimo" della Riforma. Esse si concentrano in una serie di distinzioni del tutto preziose per guadagnare una vera intelligenza di quanto è in gioco nel ruolo che ad essa si attribuisce da parte della coscienza ecclesiale contemporanea. Proviamo ad esaminare le principali: 
a) Partecizione "attraverso il rito" o "al rito"?

La prima accezione del termine si dispone nei confronti del rito con un atteggiamento sostanzialmente "strumentale", mentre la seconda accetta una esperienza rituale che non venga catturata nelle distinzioni astratte tra forma/contenuto, attivo/passivo e soggettivo/oggettivo. G. Bonaccorso vuole ricordarci, anzitutto, che si tratta di attribuire al rito una autorità originaria, una capacità fontale di istituire un rapporto più ricco e profondo con il mistero. Ciò ha evidenti ricadute nella considerazione della dimensione corporea, pratica, sensibile della liturgia.

b) Le diverse sfere di partecipazione (coscienza, azione, emozione)

I tre aggettivi che più frequentemente accompagnano il termine "partecipazione" nella stagione postconciliare sono "consapevole, attiva e pia". Ognuno di questi aggettivi riguarda una delle "facoltà" dell'uomo (conoscere, agire, sentire): la comprensione adeguata di ognuno di questi aggettivi mette in luce le possibili generalizzazioni che intellettualismo razionalistico, attivismo prassistico e devozionalismo sentimentale sono in grado di introdurre nella esperienza ecclesiale. Del tutto rilevante è il modo con cui viene intesa l'azione nell'ambito del rito:

In tale contesto, infatti, l'azione non coincide con ciò che si fa (o non si fa) durante il rito, ma nel lasciarsi fare, nel lasciarsi agire dal rito; di conseguenza, si partecipa al rito patendone l'azione...La partecipazione attiva alla liturgia è l'essere parte di quella azone rituale nella quale l'uomo è passivo perché si lascia anticipare dal sacro, dal mistero (BONACCORSO, 2009, p. 18).

c) Partecipazione comunicativa e/o simbolica

Vi è infine una distinzione importante che viene proposta tra ciò che la liturgia comunica (con un gioco delicato di codici e di messaggi) e ciò che la liturgia non può comunicare, ma solo misteriosamente accogliere, custodire, additare. "La liturgia è anzitutto il prendersi cura della parola come realtà sacra (o santa)" (BONACCORSO, 2009, p. 24). Per questo occorre tutta la competenza nel graduare i diversi livelli di comunicazione - secondo un'economia di messaggi nella ricchezza di codici - per salvaguardare la dinamica tra parola e silenzio, che resta sullo sfondo la più grande possibilità espressiva dell'atto liturgico:

Si tratta di una partecipazione simbolica nel senso stretto del termine: una partecipazione la cui essenza è di tenere insieme gli opposti, ossia 
il dire e l'impossibilità di dire, l'esprimere e l'impossibilità di esprimere, l'imaginare e l'impossibilità di immaginare, il comunicare e l'impossibilità di comunicare (BONACCORSO, 2009, p. 25).

Coniugare l'azione del parlare con la passione del tacere è la vocazione più segreta e decisiva della partecipazione alla liturgia.

Le considerazioni di Bonaccorso, qui brevemente riassunte, mettono in luce la grande ricchezza del concetto di "partecipazione", senza mai indulgere a dualismi o a soluzioni drastiche, ma richiamando alla logica corporea e simbolica della azione rituale, che opera secondo le sue "contingenze necessarie".

\section{La "partecipazione attiva" secondo J. Ratzinger}

Anche la riflessione di J. Ratzinger appare segnata da preoccupazioni di ripensamento del concetto di "actuosa participatio", pur procedendo secondo categorie e argomentazioni molto diverse, in certo senso più "classiche", e che per questo risultano meno sensibili alla novità della questione liturgica, in tutta la sua urgenza. Infatti, se anch'egli appare preoccupato delle derive intellettualistiche e prassistiche che si sono volute far discendere dalla nozione di "partecipazione attiva", tuttavia ne recupera il vero senso con un percorso biblico-liturgico che risale alla "sola vera actio", ossia al "canone eucaristico", alla "oratio": in essa, proprio per la sua qualità di azione nel senso più alto "accade che l'actio umana (così come è stata esercitata dai sacerdoti nelle diverse religioni) passa in secondo piano e lascia spazio all'actio divina, all'agire di Dio" (RATZINGER, 2001).

Di qui Ratzinger deduce che la vera azione della liturgia è l'azione di Dio, rispetto a cui tutto il resto appare secondario. Ma proprio qui, in questa estrema sintesi della argomentazione, nell'apparire del classico argomento "riduzionistico" che si presenza con la espressione "non è altro che...", riappaiono con grande forza le coppie concettuali che Bonaccorso pone in discussione, ossia le coppie sostanziale/accidentale e interiore/ esteriore. Il guadagno concettuale, propugnato dalla argomentazione stringata, sembra gravemente condizionato sul piano della concreta 
realizzazione dell'azione stessa. Proviamo a seguire l'autore in questa accelerazione delle sue categorie:

E' chiaro poi che si possono distribuire in maniera sensata le azioni esteriori: leggere, cantare, accompagnare le offerte. Tuttavia la partecipazione alla litugia della parola (leggere, cantare) deve essere distinta dalla celebrazione sacramentale vera e propria. Qui dovrebbe essere chiaro a tutti che le azioni esteriori sono del tutto secondarie. L'agire dovrebbe venire meno quando arriva ciò che conta: l'oratio. E deve essere ben visibile che l'oratio è la cosa che più conta e che essa è importante perché dà spazio all'actio di Dio (RATZINGER, 2001, p. 170).

Come è evidente, la contrapposizione tra agire dell'uomo e agire di Dio appare molto rafforzata in questa "deduzione", sulla base di una sostanziale incompatibilità tra agire e contemplare, che discende da una lettura contemplativa dell'atto rituale. Ma l'autore continua rafforzando ulteriormente il proprio argomento:

Se le singole azioni... diventano l'essenziale della liturgia e questa stessa viene degradata in un generico agire, allora viene misconosciuto il vero teodramma della liturgia, che viene anzi ridotto a parodia. La vera educazione liturgica non può consistere nell'apprendimento e nell'esercizio di attività esteriori, ma nell'introduzione all'actio essenziale che fa la liturgia, nell'introduzione, cioè, alla potenza trasformante di Dio (RATZINGER, 2001, p. 171).

Come è evidente, in questa interpretazione, la "partecipazione" risulta tutta spostata sul piano della interiorità spirituale e non riesce a valorizzare "le singole azioni". Così anche le forme di "educazione" non possono tematizzare seriamente l'esteriorità. Lo stesso autore segnala questo rischio, pur recuperando il ruolo della corporeità non già sul piano della azione rituale, ma piuttosto a livello di "esistenza quotidiana":

Proprio perché la vera azione liturgica è agire di Dio, la liturgia della fede va sempre oltre l'atto cultuale, fino a investire la quotidianità, che deve a sua volta diventare 'liturgica', servizio per il cambiamento del mondo. Dal corpo viene preteso molto più che il semplice portare in giro delle suppellettili o cose simili. Viene preteso il suo pieno impegno nella quotidianità della vita (RATZINGER, 2001, p. 171). 
Dalla interiorità spirituale alla quotidianità della vita, la logica del culto spirituale procede senza che l'azione rituale in quanto tale sia mai considerata mediazione significativa: questa lettura della partecipazione alla liturgia, con tutta la sua autorevolezza, non sembra recepire le istanze del Movimento Liturgico, anzi, in qualche modo sembra addirittura smentirle e ridimensionarle. Sarebbe naturale domandarsi: è possibile che le cose stiano davvero così? La domanda sulla possibilità è tuttavia letteralmente scavalcata dalla realtà. Sono gli atti concreti - dello stesso papa Benedetto XVI - a confermare tutti i limiti di questa comprensione della "actuosa participatio", fino alla paradossale conseguenza di un "duplice regime partecipativo", di fatto istituito con il "Motu proprio" "Summorum Pontificum", nel luglio 2007, che garantisce la "unità del rito romano" nonostante "le singole azioni" risultino in molti casi vistosamente diverse. D’altra parte il rimedio è facile: basta chiudere gli occhi, e le differenze non si vedono più.

\section{Conclusioni}

\section{verso una nuova "ratio religiose sentiendi et agendi"}

La "dottrina" della "partecipazione attiva" è dunque strategica in rapporto alla Riforma Liturgica per tre grandi motivi:

a) perché ha in sé la ragione della stessa Riforma, che è soltanto atto funzionale e di servizio ad una possibile nuova partecipazione ai riti. La partecipazione attiva non è solo ciò che "successivamente" può essere realizzato con i nuovi riti, ma è la "ratio" in base alla quale si è proceduto alla Riforma. Tale "ratio", tuttavia, non può essere esplicata solo "teologicamente": essa è una modalità del "sentire" e dell' "agire" religiosamente, che può essere compresa, illustrata e favorita secondo percorsi formativi complessi, irriducibili a evidenze - o peggio a "essenze" - di carattere concettuale.

b) perché suppone una acquisita nuova nozione e funzione dei riti nella vita della chiesa. Il primo passaggio non può avvenire se non si 
chiarisce che è il "rito" in quanto tale a dover essere chiarito nella sua nozione e nella sua funzione. Una nuova definizione di rito in rapporto alla identità cristiana è dunque il secondo presupposto perché la "actuosa participatio" non sia soltanto una "nuova retorica" che lascia immutata l'esperienza ecclesiale. E' vero che una nuova e più ampia definizione di rito impegna la chiesa in una revisione profonda e parallela delle proprie pratiche come anche delle proprie teorie.

c) perché impone un diverso modo di comprendere il nesso tra teologia e rito. Ossia rende evidente il fatto che, come non basta soltanto un aggiornamento antropologico e/o sociologico per avere migliore intelligenza dei riti, così non è sufficiente una trascrizione teologica delle nuove istanze per venire a capo della "questione liturgica". Occorre che si comprenda in modo nuovo, profondo e fecondo il lavoro teologico del rito e intorno al rito. Questo terzo fronte è forse il più impegnativo, ma anche quello che certamente risulta risolutivo per una adeguata esperienza ecclesiale della liturgia.

Proprio per questi tre motivi se si vuole ritenere che la Chiesa, la teologia e il rito debbano restare quelli di "prima", ci si deve accollare anzitutto la contestazione di questo concetto nuovo di partecipazione. Per difenderlo, tuttavia, occorre svolgere su questi tre livelli tutti gli accurati passaggi (lessicali e canonici), argomentativi e istitutivi, d'intelletto che comprende e di fede che crede, senza trascurare mai il corpo che sente. Il nostro tema della "partecipazione", dunque, comporta niente di meno che una riformulazione di tutto il modo di sentire e di agire in ambito religioso. In altri termini, il nostro concetto di "participatio actuosa" costringe ad un ripensamento complessivo della sensibilità e dell'esperienza religiosa del soggetto e non può essere ridotto ad un espediente di natura eminentemente empirica, con cui la Chiesa aggiorna il proprio rapporto con il rito (DOSSETTI, 2002, p. 82). Per usare le parole di SC, con cui vorrei concludere, esso ha invece la non piccola aspirazione di trasformare "huius nostri temporis universam rationem religiose sentiendi et agendi" (SC 43). 


\section{Riferimenti}

BLONDEL, M. L'azione: saggio di una critica della vita e di una scienza della prassi. Cinisello Balsamo: Paoline, 1993.

BONACCORSO, G. I principali orientamenti dello studio della liturgia. In: CARR, E. (Ed.). Liturgia opus trinitatis: epistemologia litúrgica. Roma: Pontificio ateneo S. Anselmo, 2002. p. 95-121. (Studia Anselmiana 133, Analecta liturgica, 24).

BONACCORSO, G. Partecipare: aspetti antropologici e teologici. In: CENTRO DI AZIONE LITURGICA - CAL (Ed.). Celebrare per avere parte al mistero di Cristo: la partecipazione alla liturgia. Roma: CLV - Edizioni Liturgiche, 2009.

BUGNINI, A. La riforma liturgica (1948-1975). Roma: CLV - Edizioni Liturgiche, 1997.

BUX, N. La riforma di Benedetto XVI. Casale Monferrato: Piemme, 2008.

CARDITA, A. Actuosa participatio: reflexão à volta de uma noção chave na "questão litúrgica". Humanística e Teologia, v. 25, p. 87-104, 2004.

CATELLA, A. Dalla Costituzione conciliare "Sacrosanctum Concilium" all'enciclica "Mediator Dei”. In: AA. VV. La mediator dei e il centro di azione liturgica: 50 anni alla luce del movimento liturgico Roma: CLV - Edizioni Liturgiche, 1998. p. 11-43. (Bibliotheca "Ephemerides Liturgicae" - Sectio Pastoralis, 18).

DE CLERCK, P. La participation active: perspectives historico-liturgiques de Pie X à Vatican II. Questions Liturgiques, v. 85, p. 11-29, 2004.

DOSSETTI, G. Per una chiesa eucaristica: rilettura della portata dottrinale della Costituzione liturgica del Vaticano II. Lezioni del 1965. Bologna: Società editrice il Mulino, 2002.

GRILLO, A. La partecipazione attiva come superamento del "paradigma medioevale" nella comprensione della ministerialita' sacramentale. In: CALAPAJ, A. M. (Ed.). Liturgia e ministeri ecclesiali. In: SETTIMANA DI STUDIO DELLA ASSOCIAZIONE PROFESSORI DI LITURGIA, 35., 2008, Roma. Atti della... Roma: CLV - Edizioni Liturgiche, 2008. p. 159-185. 
LERCARO, G. A Messa, figlioli! Direttorio liturgico per la partecipazione attiva dei fedeli alla Santa Messa letta. Bologna: EDB, 1955.

LERCARO, G. L'eucaristia nelle nostre mani: liturgia e catechesi. Bologna: EDB, 1968.

PARSCH, $\mathrm{P}$. Le renouveau liturgique au service de la paroisse: sens et portée de la liturgie populaire. Mulhouse: Salvator, 1950.

RATZINGER, J. Introduzione allo spirito della liturgia. Cinisello Balsamo: San Paolo, 2001.

REDTENBACHER, A. Pius Parsch in der Liturgiewissenschaftlichen Rezeption: Veröffentlichte und Unveröffentlichte Arbeiten über Pius Parsch. Heiliger Dienst, v. 58, p. 142-167, 2004.

Ricevuto: 20/01/2012

Recebido: 20/01/2012

Approvato: 04/02/2012

Aprovado: 04/02/2012 